\title{
Brugada syndrome coinciding with fever and pandemic (H1N1) influenza
}

\author{
Adrian Baranchuk MD, Christopher S. Simpson MD
}

Competing interests:

Dr. Baranchuk has received honoraria from Medtronic and St. Jude Medical. No competing interests

declared by Christopher

Simpson.

This article has been peer reviewed.

\section{Affiliation: From the} Kingston General Hospital, Queen's University, Kingston, Ont.

Correspondence to: Dr. Adrian Baranchuk, barancha@kgh.kari.net

CMAJ 2011. DOI:10.1503 /cmaj.100016 Arrhythmia Service,

$\Lambda$ 49-year-old man was admitted for investigation of recurrent syncopal episodes that had begun during an infection with pandemic (H1N1) 2009 virus and had continued during a postinfluenza febrile pneumonia. His family history was significant for the sudden, unexplained death of his brother at 33 years of age. A 12-lead electrocardiogram (ECG) (Figure 1), recorded while the patient was febrile (temperature $38.3^{\circ} \mathrm{C}$ ), showed ST-segment elevation in leads V1-V3, consistent with a type-I (coved) Brugada ECG pattern. A second ECG (Appendix 1, available at www.cmaj.ca/cgi/content/full/cmaj $.100016 / \mathrm{DC} 1)$, recorded after the fever had resolved, showed normal ventricular repolarization. During a subsequent electrophysiology study, no ventricular arrhythmias were induced. A procainamide challenge test resulted in the dynamic induction of a type-I Brugada ECG pattern. Because of these findings, together with his history of recurrent syncope and family

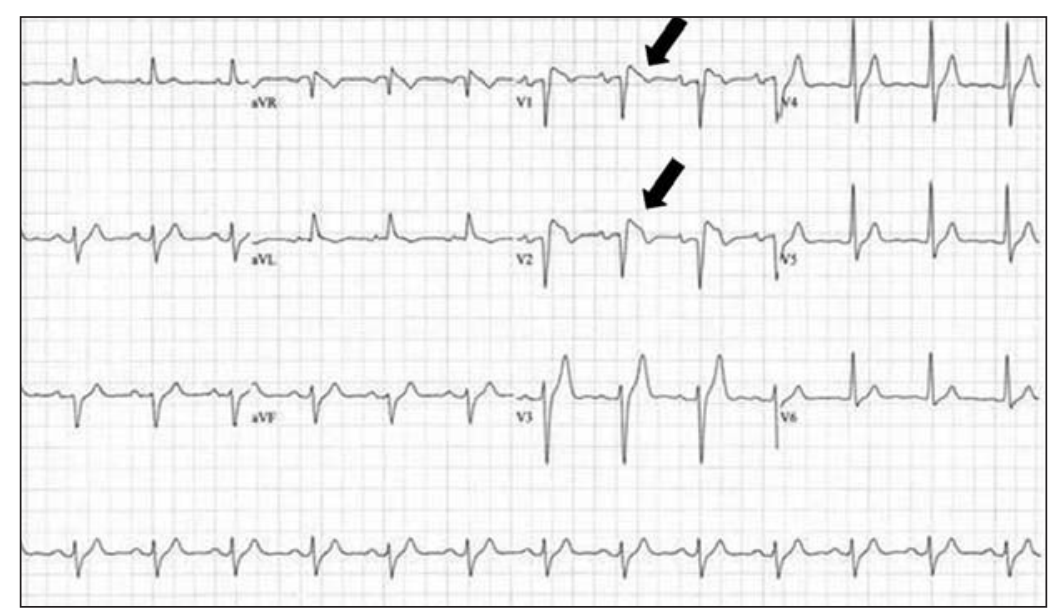

Figure 1: A 12-lead electrocardiogram (ECG) of a 49-year-old man with fever who was admitted to hospital for investigation of recurrent syncopal episodes. ST-segment elevation is obvious in two of the right precordial leads (V1-V3) (arrows), consistent with a type I (coved) Brugada ECG pattern. history of sudden, unexplained death, the patient was given an implantable cardioverter defibrillator (Medtronic, Virtuoso DR). Subsequent genetic testing, though not essential to the diagnosis, identified an SCN5A mutation, consistent with Brugada syndrome. Electrocardiogram screening and possible procainamide challenge testing was planned for consenting family members.

Brugada syndrome accounts for up to $20 \%$ of sudden cardiac deaths in patients with structurally normal hearts. ${ }^{1-3}$ It predisposes to malignant ventricular arrhythmias and can be unmasked by certain medications and by physiologic stress, including fever. ${ }^{2}$ The ECG manifestations of Brugada syndrome include a distinct ST-segment elevation in the right precordial leads; the syndrome can be manifest (apparent without pharmacological or other physiologic challenge) or concealed. ${ }^{1}$ The true prevalence of each form of the syndrome is difficult to estimate given that most patients with the syndrome are asymptomatic. Patients with Brugada syndrome (classic ECG pattern, manifest or concealed types, plus a history of syncope or aborted sudden cardiac death), are at high risk for sudden arrhythmic death, and implantable cardioverter defibrillators are the only accepted therapy to prevent sudden cardiac death. ${ }^{3}$ It remains controversial whether implantable cardioverter defibrillators should be used in patients with an asymptomatic Brugada ECG pattern.

\section{References}

1. Brugada P, Brugada J. Right bundle branch block, persistent ST segment elevation and sudden cardiac death: a distinct clinical and electrocardiographic syndrome. A multicenter report. $J$ Am Coll Cardiol 1992;20:1391-6.

2. Dumaine R, Towbin JA, Brugada P, et al. Ionic mechanisms responsible for the electrocardiographic phenotype of the Brugada syndrome are temperature dependent. Circ Res 1999;85: 803-9.

3. Antzelevitch C, Brugada R, Borggrefe M, et al. Brugada syndrome. Report of the Second Consensus Conference. Circulation 2005;111:659-70. 